\title{
Coupling light from a high-Q microsphere resonator using a UV-induced surface grating
}

\author{
V. S. Ilchenko', D. S. Starodubov ${ }^{2}$, M. L.Gorodetsky ${ }^{3}$, L. Maleki ${ }^{1}$, J. Feinberg ${ }^{2,4}$ \\ 1 Jet Propulsion Lab 298-100, 4800 Oak Grove'Drive, Pasadena, CA $91109-8099$ \\ Time and Frequency Sciences and Technology Group, Section 331, \\ (818)354-8485, FAX (818)393-6773, E-mail: ilchenko@horology.jpl.nasa.gov \\ ${ }^{2}$ D-STAR Technologies, Inc., $72533^{\text {rd }}$ St., Manhattan Beach, CA 90266 \\ ${ }^{3}$ Physics Department, Moscow State University, Moscow 119899 Russia \\ ${ }^{4}$ Department of Physics, University of Southern California, Los-Angeles, CA 90089-0484
}

High-Q microspheres with whispering-gallery modes have very narrow resonances that can be used for fiber-optic filters, ultra-compact narrow-linewidth lasers and optical/microwave oscillators $[1,2]$. Whispering-gallery modes were previously excited in microspheres using evanescent optical fields. The necessary phase synchronism was obtained by adjusting the incident angle of input light beam (prism coupler) or adjustment of the waveguide propagation constant (fiber taper coupler). For many applications, however, bulky near-field couplers are undesirable. They compromise the symmetry and generate stray fields. Also, the control of coupling is crucial for the performance of microsphere resonators: in analogy with radio frequency circuits [3] the loading Q-factor should be less than the intrinsic $Q$-factor, $Q_{L} \leq Q_{o}$ [3]. Ideally one should combine a stable coupling element and a resonator into a single microsphere component.

In this report we demonstrate, for the first time to our knowledge, far-field coupling of light from a microsphere resonator via a refractive-index grating written onto the sphere's surface. The grating is written by shining a pattern of UV light onto the surface doped with germanium to increase its photosensitivity [4].

Fused silica spheres were made by fusing a preform in a miniature flame. A 3-5 micron thick photosensitive layer was then deposited by melting germanium-oxide doped glass powder. The qualityfactor of the resulting spheres without any surface grating was $Q_{0}=(1-2) \times 10^{8}$ at $\lambda=1550 \mathrm{~nm}$, and was the same for spheres without the photosensitive layer. Surface gratings were then fabricated using $40 \mathrm{~mW}$ of 244-nm UV light from a frequency-doubled Ar laser. The exposure time was 5-10 min. The expected index modulation was $\sim(1-3) \times 10^{-4}$. The grating period was $\sim 2 \mu \mathrm{m}$ and the grating length was $\sim 15 \mu \mathrm{m}$. The period of the grating was selected to provide first-order phase matching of a whispering-gallery mode and a free-space beam oriented at $\sim 45$ degrees to the microsphere's surface.

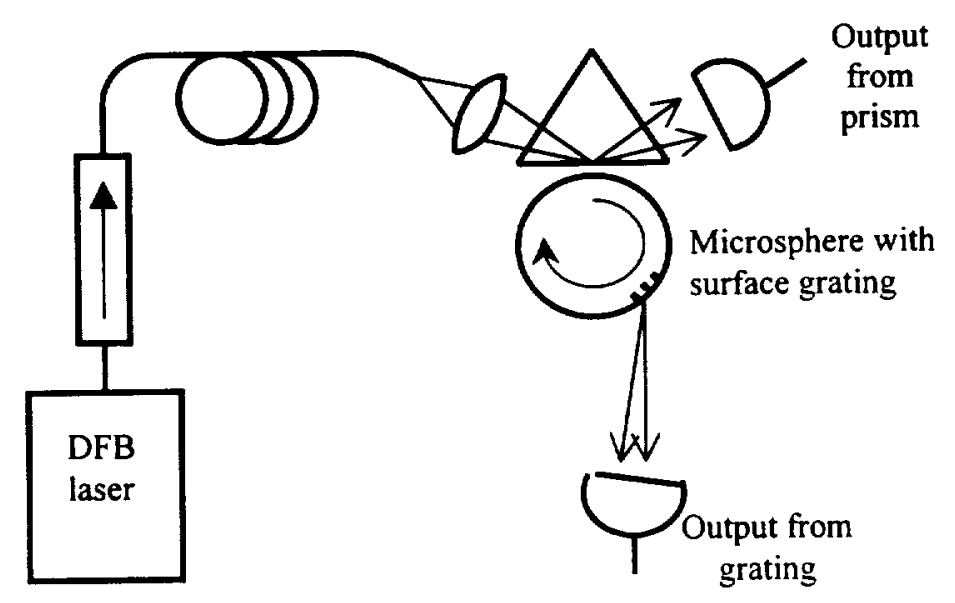

Fig.1. Experimental setup for measuring grating coupling from a microsphere resonator. 


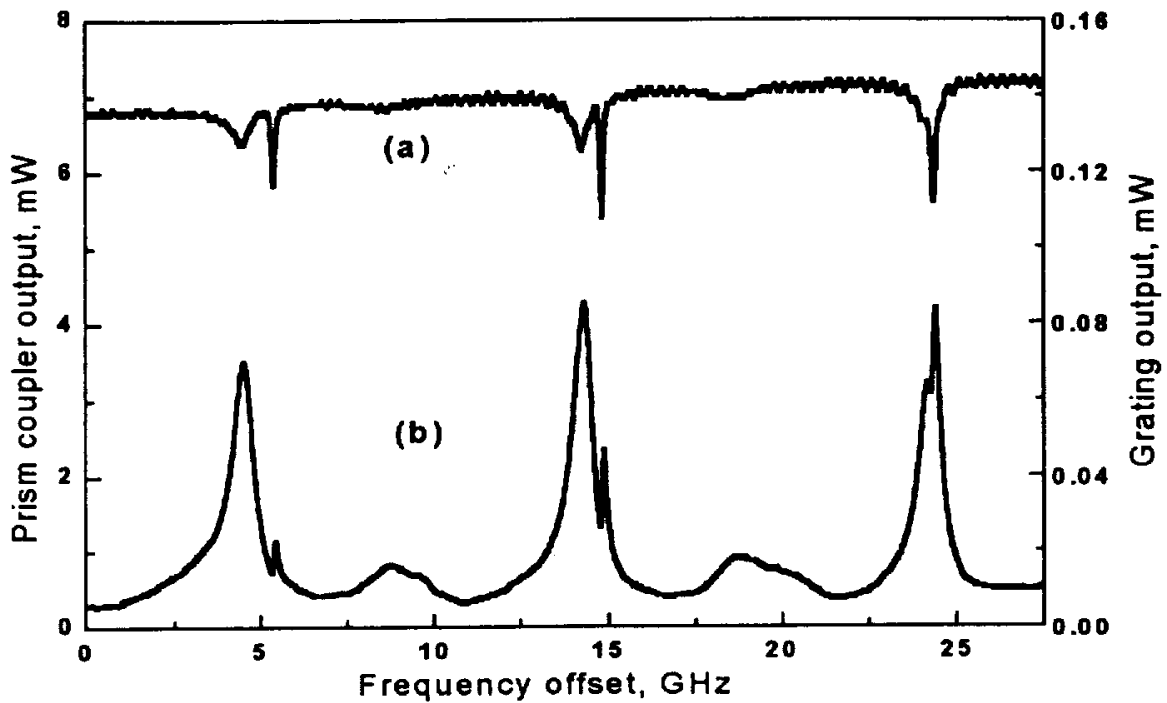

Fig.2. Whispering-gallery mode spectra obtained with a prism incoupler and a grating out-coupler. (a) Light depleted from the input beam at the whispering gallery mode resonances. (b) Light emitted from the sphere's surface grating. The maximum grating coupling efficiency was $14 \%$. The grating strength was enough to load the $Q$-factor to $(0.2 \ldots 2) \times 10^{6}$ (depending on the mode) from an initial $Q$ of $1.2 \times 10^{8}$ with no grating. Wavelength $=$ $1550 \mathrm{~nm} ;$ microsphere diameter $=185 \mu \mathrm{m}$.

Probe light from a tunable 1550-nm DFB laser was coupled into whispering-gallery modes using the standard prism-coupling technique (Fig. 1), and then coupled out of the sphere by the grating. Figure 2a shows that light was depleted from the incident beam and entered the sphere when the laser's frequency hit $a$ whispering mode resonance. Figure $2 b$ shows that the in-coupled light was then out-coupled by the grating into a free space beam that hit the second detector. The beam from the grating had $80 \%$ of its power in a single lobe, was oriented at about 40 degrees to the surface, and had a FWHM divergence of 11 degrees. From Fig.2 we derive that the grating is sufficient to load the cavity down to $Q \cong \mathrm{Q}_{\mathrm{L}} \sim 10^{5}-10^{6}$, with a maximum demonstrated coupling efficiency (ratio of grating output to total injected power in the mode) of $14 \%$. Higher $Q$ factors for a microsphere resonator with grating couplers can be obtained by simply reducing the grating strength. Efficiency of the coupling can be increased by optimizing the UV exposure, improving the grating profile, and minimizing the associated scattering losses. Parasitic coupling to low-Q higher-order modes inside the sphere can be avoided by using a smaller diameter sphere.

In conclusion, we have demonstrated a novel and flexible technique for coupling light between the whispering-gallery modes of a microsphere resonator and a free space beam.

[1] V.B.Braginsky, M.L.Gorodetsky, and V.S.Ilchenko, Phys.Lett. A 137, 393(1987); L.Collot, V.LefevreSeguin, M.Brune, J.-M.Raimond, and S.Haroche, Europhys.Lett. 23 (5), 327 (1993); H.Mabuchi and H.J.Kimble, Opt.Lett. 19, 749 (1994).

[2] A.Serpenguzel, S.Arnold, and G.Griffel, Opt.Lett. 20, 654 (1994); S.Shchiller and R.L.Byer, Opt.Lett. 16, 1138 (1991);V.Sandoghdar, F.Treussart, J.Hare,V.Lefevre-Seguin, J.-M.Raimond, and S.Haroche, Phys.Rev B54, R1777 (1996); V.V.Vassiliev, V.L.Velichansky, M:L.Gorodetsky, V.S.Ilchenko, L.Hollberg, and A.V Yarovitsky, Quantum Electronics 26, 657 (1996); D.W.Vernooy, A.Furusawa, N.Ph.Georgiades, V.S.Ilchenko, and H.,J.Kimble, Phys Rev A 57, R2293 (1998).

[3] J.C.Knight, G.Cheung, F.Jacques, T.A.Birks, Opt.Lett. 22, 1129 (1997); M.L.Gorodetsky, and V.S.Ilchenko, JOSA-B 15 (1998) in press.

[4] D. S. Starodubov, V. Grubsky, J. Feinberg, B. Kobrin, and S. Juma, Opt.Lett. 22, 1086 (1997). 